\title{
Dinâmica de afetos dos alunos em um Sistema Tutor Inteligente de matemática no contexto brasileiro
}

\author{
Felipe de Morais ${ }^{1}$, Patrícia A. Jaques ${ }^{1}$ \\ ${ }^{1}$ Programa de Pós Graduação em Computação Aplicada (PPGCA) \\ Universidade do Vale do Rio dos Sinos (UNISINOS), São Leopoldo - RS - Brasil \\ felipmoraisdedu.unisinos.br, pjaques@unisinos.br
}

\begin{abstract}
Affect dynamics study how students' emotions develop and manifest during the use of computer-based learning environments. Recent research has shown that the affect dynamics is not generic and depends on the students' context, mainly their country. When analyzing the Brazilian research literature, we found that no research developed an affect dynamics model statistically validated in the Brazilian context. This work presents the first affect dynamics model, with statistical validity, using data from students learning with a step-based ITS for math. This model enabled the discovery of significant transitions between academic emotions confusion, frustration, boredom, and engagement.
\end{abstract}

Resumo. A pesquisa em dinâmica de afetos estuda como as emoções dos alunos se desenvolvem e se manifestam durante a aprendizagem. Trabalhos recentes mostraram que essa dinâmica não é genérica e que depende do contexto dos alunos, principalmente seu país de origem. Identificamos que nenhuma pesquisa desenvolveu um modelo de dinâmica de afetos validado no contexto brasileiro. Este trabalho apresenta o primeiro modelo de dinâmica de afetos utilizando dados de alunos brasileiros aprendendo com um STI de matemática baseado em passos. Este modelo possibilitou a descoberta de transições significativas entre as emoções acadêmicas confusão, frustração, tédio e engajamento.

\section{Introdução}

Os estados afetivos têm um papel fundamental nos alunos em sala de aula, pois podem influenciar a cognição, interferindo no processo de aprendizagem [Azevedo and Aleven 2013]. Mais especificamente, as emoções, um tipo de estado afetivo [Scherer 2005], podem influenciar a aprendizagem tanto de forma positiva quanto negativa, pois são capazes de controlar atenção, motivação, cognição, modelagem de estratégias e autorregulação da aprendizagem [Pekrun 2014]. Emoções positivas, como alegria, engajamento e curiosidade podem afetar aspectos físicos, sociais, intelectuais e criativos, influenciando positivamente no desenvolvimento destas capacidades [Fredrickson 1998]. Por outro lado, as emoções negativas, como frustração e tédio, podem afetar negativamente o processo de aprendizagem [Graesser and D'Mello 2011]. A confusão tem sido vista como um caso particular, pois pode influenciar positivamente a aprendizagem quando for apropriadamente regulada e resolvida ou negativamente, caso contrário [D'Mello et al. 2014]. Desta forma, é importante que ambientes educacionais de aprendizagem, baseados em computador, sejam capazes de detectar e adaptar suas estratégias pedagógicas de acordo com as emoções dos alunos [Arroyo et al. 2014]. 
As emoções encontradas com mais frequência em ambientes computacionais de aprendizagem são confusão, frustração, tédio e engajamento [Calvo and D'Mello 2010, D'Mello 2013], também conhecidas como emoções de aprendizagem (learningcentered emotions) [Graesser et al. 2014] ou emoções acadêmicas (academic emotions) [Pekrun 2016]. [D’Mello and Graesser 2012] desenvolveram um modelo teórico de transição entre essas emoções, conhecido como modelo de dinâmica de afetos (affect dynamics model) [Kuppens 2015]. Nesse modelo, os autores apontam que, uma vez que o aluno entra em emoções negativas, como tédio, é improvável que este aluno se recupere e volte para uma emoção positiva. De acordo com os autores, este estado 'estacionário' pode influenciar negativamente na aprendizagem e, por isso, as emoções dos alunos precisam ser reguladas com base em intervenções pedagógicas.

Para o desenvolvimento do modelo de dinâmica de afetos, [D'Mello et al. 2007a] propuseram a métrica $\boldsymbol{L}$, que tem o objetivo de identificar a probabilidade de transição entre duas emoções, considerando a probabilidade ao acaso de cada emoção. Além disso, pode ser usada em testes de significância estatística para inferir se uma transição é significativamente mais provável do que o acaso. Desde o desenvolvimento desse modelo teórico [D'Mello and Graesser 2012], muitos trabalhos vêm se baseando nesses resultados para examinar como as emoções transitam entre si durante as atividades de aprendizagem [Bosch and D'Mello 2013, Andres and Rodrigo 2014, Ocumpaugh et al. 2017]. Porém, pesquisas mais recentes têm apontado que muitos desses trabalhos aplicaram a métrica L incorretamente, levando a conclusões inválidas [Karumbaiah et al. 2018, Karumbaiah et al. 2019]. Recentemente, a pesquisa de [Karumbaiah et al. 2021] apresenta uma reanálise dos trabalhos que utilizaram a métrica L para o desenvolvimento de dinâmica de afetos. Os autores apontam que os resultados encontrados divergem do modelo teórico apresentado por [D'Mello and Graesser 2012]. Por fim, ao comparar os resultados de dados de alunos provenientes de diferentes países, os autores ainda ressaltam que o modelo de dinâmica de afetos é culturalmente dependente.

Sabendo que a transição de emoções dos alunos parece ser sistêmica [D'Mello and Graesser 2012], mas que essa dinâmica depende do contexto cultural dos alunos [Karumbaiah et al. 2021], é importante estudar a dinâmica de afetos dos alunos no contexto onde vivemos, visto que nenhuma pesquisa tenha apresentado tais resultados. Diante disto, vimos a necessidade de realizar uma análise da dinâmica de afetos no contexto brasileiro. Para isso, inicialmente realizamos uma coleta de dados com alunos utilizando PAT2Math, um Sistema Tutor Inteligente (STI) gamificado baseado em passos, que vem auxiliando centenas de alunos no Brasil com o conteúdo de equações de primeiro grau [Jaques et al. 2013, Azevedo et al. 2018, Morais and Jaques 2021]. Após, as emoções dos alunos foram capturadas através do protocolo EmAP-ML, no qual anotadores humanos treinados codificaram as emoções confusão, frustração, tédio e engajamento por meio da análise de vídeos do rosto com áudio e da tela dos alunos [Morais et al. 2019]. Por fim, as emoções dos alunos foram utilizadas para o cálculo da estatística L, considerando as quatro emoções mais vistas nesses tipos de ambientes [Calvo and D'Mello 2010, D'Mello 2013]. Nesta etapa, consideramos a métrica L ajustada, conforme proposto por [Karumbaiah et al. 2021]. Desse modo, este trabalho relata a coleta das emoções dos alunos, o método e o código fonte utilizados para o cálculo da dinâmica de afetos e os resultados desse modelo no contexto brasileiro. 


\section{Trabalhos Relacionados}

Realizamos uma busca na literatura brasileira, visando identificar as pesquisas que vêm trabalhando na área de dinâmica de afetos. Para isso, utilizamos o sistema de busca do Portal de Publicações da Comissão Especial de Informática na Educação (CEIE) ${ }^{1}$. Esse portal possui um sistema de busca em trabalhos publicados na 1) Revista Brasileira de Informática na Educação (RBIE), 2) Simpósio Brasileiro de Informática na Educação (SBIE), 3) Workshop de Informática na Escola (WIE), 4) Congresso Brasileiro de Informática na Educação (CBIE), 5) Jornada de Atualização em Informática na Educação (JAIE), 6) Workshop de Desafios da Computação Aplicada à Educação (DesafIE) e 7) Workshop on Advanced Virtual Environments and Education (WAVE2). Esse sistema de busca permite realizar pesquisas por artigos com base em palavras chaves e operadores lógicos, como $\mathrm{E}(A N D)$, OU $(O R)$ e o caractere coringa $\left(^{*}\right)$. Assim, construímos a seguinte string de busca: (transição OR transições OR transition OR dinâmic* $O R d y$ namic *) AND (emoções OR emoção OR emotion OR afeto or afetiv* OR affect) ${ }^{2}$ para contemplar a busca por trabalhos na área de dinâmica de afetos ou transição de emoções.

Ao todo, obtivemos dez resultados, com publicações entre 2014 e 2020. Desses, seis trabalhos apresentam resultados sobre a detecção das emoções. [Dantas et al. 2015b] apresentam um detector dinâmico de emoções básicas através da análise de expressões faciais por vídeo utilizando árvores de decisão. Após, em [Dantas et al. 2015a], os autores descrevem a aplicação do modelo desenvolvido na plataforma Moodle, apresentando o impacto que informações apresentadas pelo professor podem produzir no aluno. Ainda nesta área, [Gottardo and Pimentel 2018] apresentam um modelo híbrido de detecção de emoções básicas com base em análise de vídeos e análise cognitiva por meio da interação dos alunos com o sistema. Mais recentemente, [Morais and Jaques 2019b, Morais and Jaques 2019a, Morais and Jaques 2020] apresentam a detecção de emoções baseada em mineração de dados (sensor-free affect detection) e utilizam apenas dados de interação dos alunos com o sistema $(\log s)$ para a detecção. Nesse, os autores relatam a utilização de informações das transições de emoções e da personalidade dos alunos para melhorar a acurácia dos detectores de confusão, frustração, tédio e engajamento.

Os outros quatro trabalhos não apresentam um modelo de dinâmica de afetos ou de informações sobre as transições entre emoções. Mais especificamente, [Melo et al. 2017] descreve o desenvolvimento de um modelo de aluno afetivo com base em um reconhecedor de emoções baseado em vídeos. [Gonzalez and Tamariz 2014] apresenta a inferência de emoções baseado em eventos do sistema para o ensino de programação, considerando as emoções alegria, tristeza, satisfação e frustração com base em regras fixas do tipo ifelse. [Marques et al. 2017] propõem um modelo conceitual sobre pensamento computacional na educação básica seguindo a taxonomia de Bloom. E, por fim, [Corrêa et al. 2016] relatam os resultados de questionários específicos, destacando ganhos afetivos e cognitivos dos alunos com os jogos interativos aplicados a museus.

A maioria dos trabalhos relacionados ainda é baseada na teoria das emoções básicas [Ekman 1994] (resultados também vistos em outros trabalhos de

\footnotetext{
${ }^{1}$ Portal da CEIE: https://www.br-ie.org/pub/index.php/index. A análise também foi realizada na nova base -SBC OpenLib: https://sol.sbc.org.br/index.php/cbie

${ }^{2}$ Consideramos os termos da string de busca em português e inglês pois esses veículos de publicação também aceitam artigos escritos em inglês.
} 
Computação Afetiva no Brasil [Morais et al. 2017]). Porém, já se sabe que essas emoções possuem pouca relevância em sessões de alunos interagindo com ambientes computacionais de aprendizagem [D'Mello et al. 2007b, Lehman et al. 2008, Baker et al. 2010, D'Mello and Calvo 2013]. Além disso, dos dez trabalhos, apenas [Gottardo and Pimentel 2018] e [Morais and Jaques 2020] apresentam informações sobre a quantidade, probabilidade e representatividade de cada uma das transições entre emoções. Mas desses, somente [Morais and Jaques 2020] apresenta dados especificamente sobre as emoções de aprendizagem. Assim, nenhum trabalho relacionado apresentou um modelo de dinâmica de afetos validado estatisticamente no contexto brasileiro.

Ao analisar a literatura internacional, o modelo teórico de dinâmica de afetos proposto por [D'Mello and Graesser 2012], desenvolvido com base na métrica L, é o mais conhecido na área, possuindo centenas de citações, de acordo com o Google Scholar ${ }^{3}$. Nesse modelo, traduzido na Figura 1.A, os autores apontam que alunos engajados enfrentam impasses durante o aprendizado e vivenciam confusão causada por um desequilíbrio cognitivo. Se essa confusão for regulada, os alunos se engajam novamente e aprendem com essa experiência. Caso contrário, essa confusão torna-se uma frustração, na qual o aluno fica bloqueado, sem saber como proceder. Novamente, se nenhuma intervenção for realizada, o aluno enfrentará o tédio, perdendo a esperança de aprender.

De acordo com os resultados apresentados por [D'Mello and Graesser 2012], existem dois principais aspectos que devem ser considerados desse modelo: $i$ ) o desiquilíbrio cognitivo $^{4}$ e a confusão desempenham um papel central na aprendizagem dos alunos. Assim, os autores afirmaram que os ambientes de aprendizagem devem desafiar os alunos a suscitar o pensamento crítico e a investigação profunda; $i i)$ Emoções negativas, como frustração e tédio, precisam ser reguladas com base em intervenções pedagógicas. Portanto, os autores concluem que o ambiente de aprendizagem precisa detectar esses estados para responder e adaptar suas estratégias para engajá-los novamente.

Recentemente, [Karumbaiah et al. 2021] realizaram um levantamento dos trabalhos que aplicaram a métrica L incorretamente e fizeram uma reanálise dos dados desses trabalhos considerando uma versão ajustada da métrica L. Os autores descrevem que os resultados dessa reanálise diferem significativamente dos resultados previamente reportados nos artigos primários. Além disso, os autores também apontam que poucos trabalhos relataram as mesmas transições reportadas no modelo teórico de dinâmica de afetos de [D'Mello and Graesser 2012], conforme mostra o modelo deles, traduzido na Figura 1.B.

Na Figura 1.B, o modelo Todos os dados foi desenvolvido considerando os dados de todos os trabalhos. O modelo Estados Unidos considera apenas os dados obtidos nos Estados Unidos e o modelo Filipinas considera apenas os dados obtidos nas Filipinas. As flechas verdes indicam transições significativamente prováveis, as vermelhas indicam transições significativamente improváveis e as pretas indicam as transições nulas. Por fim, um '*' azul indica as transições hipotéticas propostas por [D’Mello and Graesser 2012].

O trabalho de [Karumbaiah et al. 2021] apresenta uma análise detalhada e atual do

\footnotetext{
${ }^{3} 637$ citações, segundo https: / / scholar. google. com. br, em 2 de Setembro de 2021.

${ }^{4}$ De acordo com [D’Mello and Graesser 2012], “desequilíbrio cognitivo é um estado de incerteza que ocorre quando um indivíduo é confrontado com obstáculos aos objetivos, interrupções de sequências de ação organizadas, impasses, contradições, eventos anômalos, dissonância, incongruências, feedback inesperado, incerteza, desvios das normas e novidade."
} 


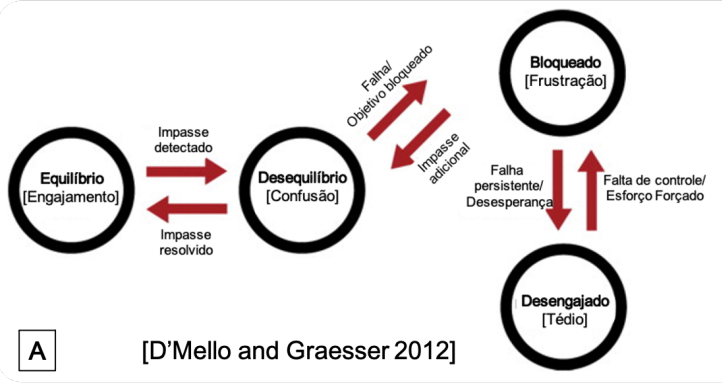

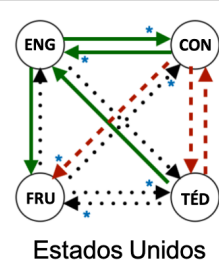
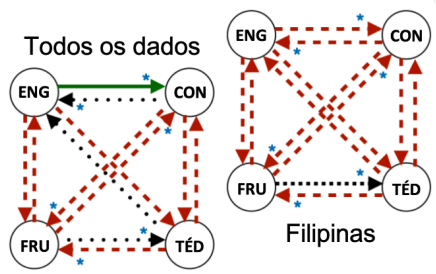

[Karumbaiah et al. 2021]

Figura 1. Modelo de dinâmica de afetos de [D'Mello and Graesser 2012] (A) e [Karumbaiah et al. 2021] (B).

estado da arte sobre pesquisas em dinâmica de afetos a nível internacional ${ }^{5}$. Assim, neste trabalho partimos dos achados e dos trabalhos relacionados já reportados pelos autores. Com base nesses resultados e na busca realizada neste trabalho na literatura brasileira, podemos delinear as seguintes conclusões: $i$ ) o modelo de dinâmica de afetos descrito por [D’Mello and Graesser 2012] não é generalizável para todos os casos, ii) o contexto, principalmente o país de origem de coleta dos dados dos alunos, pode influenciar significativamente no modelo, e iii) nenhuma pesquisa desenvolveu o modelo de dinâmica de afetos, com significância estatística, no contexto brasileiro. Desse modo, este trabalho tem como objetivo desenvolver o primeiro modelo de dinâmica de afetos considerando as emoções de alunos utilizando um STI de matemática no contexto brasileiro.

\section{Método}

Para o desenvolvimento do modelo de dinâmica de afetos, foi necessário realizar a coleta das emoções dos alunos. Essas emoções foram organizadas em ordem cronológica, para que fosse possível contabilizar cada transição de uma emoção para outra, de cada um dos alunos. Posteriormente, o cálculo da métrica L e a aplicação dos resultados obtidos às análises estatísticas foram realizados para a identificação das transições significativas.

\subsection{Coleta de dados}

Realizamos uma coleta de dados com 55 alunos, com idade entre 12 e 13 anos (média de 12,15 anos) de duas turmas do sétimo ano do ensino fundamental de uma escola privada na grande Porto Alegre. Esses alunos utilizaram o STI PAT2Math durante 10 sessões no laboratório da escola, uma vez por semana, com duração média de 40 minutos por sessão. As tarefas realizadas pelos alunos foram resolução de equações de primeiro grau, com auxílio constante do STI, que fornece feedback mínimo (indicando se o passo está certo ou errado) e também pode prover dicas especializadas, caso o estudante erre o passo ou não saiba como prosseguir. Durante as sessões, alguns alunos (selecionados aleatoriamente) tiveram seus rostos filmados com uma webcam, também capturando o áudio. De forma simultânea, esses mesmos alunos tiveram a tela de seus computadores gravadas. Ao todo, 230 vídeos completos (rosto + áudio + tela do computador) foram obtidos durante as sessões de coleta de dados. Todos os alunos participantes dessa coleta entregaram o Termo de Consentimento Livre e Esclarecido (TCLE) (validado pelo Comitê de Ética da universidade dos autores) assinado por um responsável.

\footnotetext{
${ }^{5}$ [Karumbaiah et al. 2021] apresenta uma análise atual do estado da arte, a qual não identificou nenhum trabalho sobre a dinâmica de afetos no contexto brasileiro publicado internacionalmente.
} 
Três anotadores humanos, treinados de acordo com o protocolo EmAP-ML, codificaram 30 vídeos completos. O protocolo EmAP-ML [Morais et al. 2019] possui fases de treinamento e teste de codificadores e permite a anotação das emoções dos alunos em clipes de cinco segundos com base na análise de vídeos, contendo informações do rosto com áudio sincronizados com a tela do computador do aluno. Os três anotadores, sendo dois doutorandos (incluindo o primeiro autor deste artigo) e uma pesquisadora (segunda autora do artigo) na área de computação afetiva aplicada à educação, atingiram um índice de concordância Kappa médio de 0,7 na fase de teste do protocolo. Assim, um total de 2099 rótulos de emoções foram obtidos, provenientes de 30 alunos diferentes, sendo eles 1118 rótulos de engajamento (53,3\%), 463 de confusão (22,1\%), 90 de tédio $(4,3 \%)$ e 51 de frustração (2,4\%). Além disso, 377 rótulos foram classificados como outro, que identifica um estado diferente desses quatro ou um estado não identificado pelos codificadores.

\subsection{Métrica $\mathbf{L}$}

[D’Mello et al. 2007a] propuseram a métrica $\mathbf{L}$ para calcular a probabilidade de transição entre duas emoções. Essa métrica é adequada porque aborda a influência da taxa base (frequência proporcional de uma única emoção), penalizando associações que não são maiores do que uma quantidade esperada de associação. Dada uma sequência de emoções, essa equação calcula a probabilidade de que uma emoção (anterior) transite para uma emoção subsequente (próxima), considerando a taxa base da próxima emoção.

De acordo com [D’Mello et al. 2007a], L maior do que 0 indica maior probabilidade de transição, $\mathrm{L}$ menor do que 0 indica menor probabilidade de transição e $\mathrm{L}$ igual a zero indica probabilidade ao acaso ${ }^{6}$. O problema metodológico, reportado por [Karumbaiah et al. 2018, Karumbaiah et al. 2019, Karumbaiah et al. 2021], que afetou os resultados de várias pesquisas, se refere ao pré-processamento das emoções. De acordo com os autores, quando as transições de um estado para ele mesmo (self-transitions) são desconsideradas, o valor de $\mathrm{L}$ que define a probabilidade ao acaso deve ser ajustado. Assim, os autores propuseram um ajuste para esse valor, seguindo a fórmula $1 /(n-1)^{2}$, onde $n$ é o número de emoções consideradas. Neste trabalho, além das quatro emoções de aprendizagem (confusão, frustração, tédio e engajamento), nós também consideramos a transição para o estado outro, totalizando $n$ igual a cinco. Desse modo, ao invés de considerar L igual a 0 para a probabilidade ao acaso, nós consideramos L igual a 0,0625.

\subsection{Desenvolvimento do modelo de dinâmica de afetos}

Uma vez coletados os rótulos de emoções, realizamos uma etapa de pré-processamento para organizar e estruturar os dados obtidos para o desenvolvimento do modelo de dinâmica de afetos. Primeiro, separamos os dados de cada aluno e ordenamos os rótulos das emoções de acordo com a sua ordem de captura, realizada pelos codificadores. Depois, importamos os dados para o Google Colab $^{7}$, permitindo o desenvolvimento de um script em Python para a $i$ ) leitura e pré-processamento dos dados, ii) cálculo da métrica L, iii) aplicação dos métodos estatísticos, iv) geração dos resultados em forma tabular e v) impressão do gráfico de dinâmica de afetos apenas com as transições significativas.

\footnotetext{
${ }^{6}$ A probabilidade ao acaso é calculada de acordo com a quantidade de emoções consideradas. Assim, independente da emoção que o aluno está sentindo, a probabilidade de ir para uma outra emoção é a mesma para todas as emoções consideradas. Ou seja, 1 dividido pelo número total de emoções consideradas.

${ }^{7}$ https: / / colab.research.google.com/notebooks/welcome.ipynb?hl=pt-BR
} 
Para calcular a métrica L, utilizamos o código previamente disponibilizado por [Matayoshi et al. 2020]. Nesse código, os autores já realizam um processo de remoção de autotransições (self-transitions), eliminando todas as transições de uma emoção para ela mesma na lista de transições de emoções de cada aluno. Após, a métrica L é calculada para cada permutação de transição entre emoções, gerando uma matriz de valores L. Depois, realizamos a aplicação do teste $t$ para calcular a significância estatística das transições obtidas considerando todos os alunos. Além disso, também aplicamos o procedimento de correção post-hoc de Benjamini-Hochberg $(\mathrm{BH})$ para controlar os resultados falso-positivos (usando um $\alpha$ de 0,05), uma vez que o conjunto de hipóteses envolve múltiplas comparações. Este procedimento estatístico foi seguido de acordo com pesquisas que vêm trabalhando com a dinâmica de afetos [Karumbaiah et al. 2021].

Por fim, após obter todos os resultados da métrica L de cada combinação de transição entre emoções e a validação estatística das transições significativas, realizamos a impressão dos resultados em forma tabular e a geração automática do gráfico de dinâmica de afetos. A impressão dos resultados foi desenvolvida de forma adaptável, permitindo a exibição de diferentes informações e de fácil exportação, seguindo o formato CSV. Já a geração do grafo de dinâmica de afetos considera os valores L e a significância estatística reportada pelo método post-hoc BH para cada transição. Assim, somente as transições estatisticamente significativas são exibidas. O código desenvolvido para o cálculo de dinâmica de afetos deste trabalho está disponível online ${ }^{8}$.

\section{Resultados e Discussões}

Esta seção descreve os resultados do modelo de dinâmica de afetos no contexto brasileiro e apresenta discussões em relação aos mesmos. A Tabela 1 descreve o resultado da métrica L para cada uma das transições, indicando a probabilidade de ir para uma emoção (próxima) já estando em uma determinada emoção (anterior). A Tabela 1 apresenta os resultados para as emoções engajamento $(\mathrm{ENG})^{9}$, confusão $(\mathrm{CON})$, frustração (FRU), tédio (TED) e outro (OUT). O primeiro valor dentro das células da tabela indica o resultado da métrica L. O segundo valor, entre parênteses, representa o valor $p$ ajustado de acordo com o método post-hoc HB. O terceiro valor, entre colchetes, exibe a quantidade de transições entre a emoção anterior e a próxima. Ainda, os valores destacados em negrito indicam que a transição é significativa, de acordo com o método post-hoc HB.

Tabela 1. Resultados estatísticos da dinâmica de afetos no contexto brasileiro.

\begin{tabular}{|c|c|c|c|c|c|}
\hline Anterior $\backslash$ Próxima & ENG & CON & FRU & TED & OUT \\
\hline ENG & - & $0,2761(0,0000)[123]$ & $0,0068(0,0000)[16]$ & $0,0139(0,0054)[25]$ & $0,2650(0,0000)[120]$ \\
\hline $\mathrm{CON}$ & $0,5002(0,0000)[116]$ & - & $0,0364(0,2876)[13]$ & $-0,0270(0,0002)[7]$ & $-0,0397(0,0809)[35]$ \\
\hline FRU & $0,0420(0,8937)[12]$ & $0,1747(0,3287)[11]$ & - & $-0,0375(0,0238)[1]$ & $-0,0177(0,4719)[9]$ \\
\hline TED & $\mathbf{0 , 4 7 8 8}(0,0008)[31]$ & $-0,2082(0,0000)[4]$ & $0,0471(0,7927)[2]$ & - & $-0,0766(0,0661)[11]$ \\
\hline OUT & $0,5670(0,0000)[119]$ & $-0,1001(0,0020)[32]$ & $-0,0341(0,0000)[2]$ & $0,0317(0,1639)[17]$ & - \\
\hline
\end{tabular}

Para facilitar o entendimento, podemos pegar o exemplo da linha três, coluna dois. Nesse exemplo, a Tabela 1 exibe a estatística da transição de confusão (anterior) para engajamento (próxima). O primeiro valor, $L=0,5002$, indica que existe uma alta probabilidade de um aluno confuso ir para o estado de engajamento. O segundo valor, valor $p<0,05$, indica que essa é uma probabilidade significativa, ou seja, com evidência

\footnotetext{
${ }^{8}$ https://github.com/felipmorais/Calculo_da_dinamica_de_afetos

${ }^{9}$ As emoções foram abreviadas para facilitar a visualização no gráfico apresentado na Figura 2.
} 
estatística. Por fim, o terceiro valor, em colchetes, exibe que o número de transições que ocorreram partindo de confusão para engajamento é igual a 116. Ao todo, calculamos 706 transições, não considerando as autotransições (self-transisions).

A Figura 2 ilustra o gráfico de dinâmica de afetos, no qual somente as transições significativas são exibidas. No gráfico, as flechas azuis representam maior probabilidade e as vermelhas representam menor probabilidade de transição. O número próximo à ponta da flecha indica o valor L para a transição sendo exibida e a espessura da linha é proporcional a esse valor, sendo que quanto maior o valor de L, mais grossa é a linha. Como este trabalho considera a métrica L ajustada, de acordo com [Karumbaiah et al. 2021], o valor de $\mathrm{L}$ ao acaso considerado foi o de 0,0625 . Ou seja, uma transição menor do que esse limiar é considerada de baixa probabilidade e, por outro lado, uma transição maior é considerada de maior probabilidade, comparando à probabilidade ao acaso.

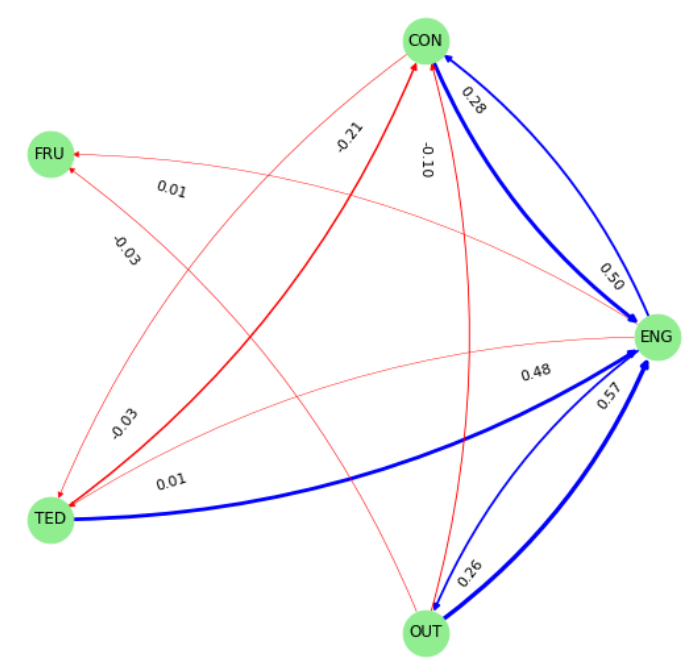

Figura 2. Modelo de dinâmica de afetos no contexto brasileiro.

Ao analisar o modelo de dinâmica de afetos desenvolvido neste trabalho, podemos notar que existe uma maior probabilidade dos alunos permanecerem em um ciclo, transitando entre os estados de engajamento e confusão. Apesar de não identificarmos nenhuma transição significativa de maior probabilidade saindo de confusão, [D’Mello et al. 2014] descrevem que a confusão é benéfica para aprendizagem quando resolvida, podendo ser prejudicial caso contrário. Podemos notar também que é improvável que o aluno transite de engajamento para frustração ou tédio. É interessante notar que mesmo sendo improvável que o aluno fique entendiado, há maior probabilidade dele estar entediado e transitar para engajado, contrariando os resultados de [D'Mello and Graesser 2012]. Estudos mostram que alunos entendiados utilizam de comportamentos fora da tarefa (offtask) para ajudá-los a se engajar novamente, tendo um impacto positivo na aprendizagem [Baker et al. 2011]. Por fim, notamos também que há uma maior probabilidade de transição de engajamento para o estado outro. Embora nenhum trabalho relacionado tenha desenvolvido o modelo de dinâmica de afetos considerando o estado outro, acreditamos que esse seja um resultado a ser considerado, possivelmente indicando a ocorrência de outras emoções que também sejam mais frequentes, além das quatro consideradas.

Ao comparar o modelo de dinâmica de afetos desenvolvido neste trabalho com o modelo teórico mais conhecido na área [D’Mello and Graesser 2012] (so- 
mente as transições significativas), podemos notar que somente as transições $E N$ Gajamento $\rightarrow$ CONfusão e CONfusão $\rightarrow$ ENGajamento são similares. Como diferenças, podemos ressaltar a transição TEDio $\rightarrow$ ENGajamento, que possui uma alta probabilidade no modelo desenvolvido, diferentemente do que é apresentado em [D'Mello and Graesser 2012]. Também comparamos o modelo desenvolvido com o modelo apresentado por [Karumbaiah et al. 2021]. Os autores desenvolveram três modelos de acordo com o país de origem dos alunos, sendo eles somente Estados Unidos, somente Filipinas e o modelo completo, com dados dos dois países. O modelo que considera somente os dados dos Estados Unidos é o mais similar com o modelo deste trabalho. Identificamos cinco transições significativas similares, sendo ENGajamento $\rightarrow$ CONfusão, CONfusão $\rightarrow$ ENGajamento, TEDio $\rightarrow$ ENGajamento, TEDio $\rightarrow$ CONfusão e CONfusão $\rightarrow$ TEDio. Como diferenças, podemos destacar a transição ENGajamento $\rightarrow$ FRUstração, que no modelo de [Karumbaiah et al. 2021] possui uma probabilidade maior do que ao acaso e no modelo deste trabalho é menor do que ao acaso. Outra transição diferente foi ENGajamento $\rightarrow$ TEDio, que no modelo de [Karumbaiah et al. 2021] é nulo, enquanto neste trabalho é significativo mas com baixa probabilidade. Como os modelos de [Karumbaiah et al. 2021] e [D'Mello and Graesser 2012] não consideram o estado OUTro, não comparamos transições ligadas a esse estado.

Ao analisar de forma mais macro o modelo de dinâmica de afetos deste trabalho com os trabalhos relacionados, podemos destacar que o modelo desenvolvido está mais alinhado com o trabalho de [Karumbaiah et al. 2021], utilizando o contexto dos alunos dos Estados Unidos como comparativo. Conforme apresentado na revisão feita pelos autores, poucas pesquisas chegaram em resultados similares ao modelo proposto por [D'Mello and Graesser 2012]. Algumas justificativas descritas na literatura sobre essa diferença se referem a coleta de dados de [D'Mello and Graesser 2012] ter sido realizada com alunos em laboratório, enquanto que os demais trabalhos coletaram os dados em ambientes reais nas escolas. Outra possibilidade está na forma de coleta de emoções, no qual [D’Mello and Graesser 2012] coletou as emoções com as anotações dos próprios alunos, de forma bem detalhada. Diferentemente, [Karumbaiah et al. 2021] utilizaram o protocolo BROMP [Ocumpaugh 2015], no qual codificadores treinados anotam as emoções dos alunos em tempo real, com um intervalo grande entre uma emoção e outra para o mesmo aluno, visto que o protocolo segue um método de rodízio, analisando um aluno por vez. Neste trabalho, utilizamos o protocolo EmAP-ML, baseado em análise de vídeos, onde anotadores treinados são capazes de coletar as emoções de forma muito detalhada, na qual é analisado um aluno por vez por um período estendido [Morais et al. 2019]. Outra diferença está no ambiente de aprendizagem, no qual este trabalho é o único a relatar a coleta de dados de um STI baseado em passos gamificado voltado ao ensino de matemática. Apesar dos diferentes métodos de coleta das emoções e ambientes nos dois trabalhos relacionados e com esse trabalho, podemos notar uma forte semelhança no modelo de dinâmica de afetos deste trabalho com [Karumbaiah et al. 2021].

\section{Conclusão}

O desenvolvimento de intervenções pedagógicas efetivas em ambientes computacionais de aprendizagem em tempo real depende do entendimento de como as emoções dos alunos se desenvolvem e se manifestam com o tempo, área conhecida como dinâmica de afetos [Kuppens 2015]. Ao analisar os trabalhos mais recentes nesta área, é possível identificar 
que a dinâmica de afetos não é genérica para todos os casos e é dependente de contexto, tais como o país em estudo, o ambiente de aprendizagem empregado, as características dos estudantes, entre outros. Realizamos uma análise na literatura buscando por trabalhos na área de dinâmica de afetos considerando dados de estudantes brasileiros. Porém, não encontramos trabalhos que desenvolveram um modelo de dinâmica de afetos com validade estatística para o contexto brasileiro.

Este trabalho traz duas contribuições para a comunidade de informática de educação no Brasil. A primeira delas é o desenvolvimento do primeiro modelo de dinâmica de afetos com validade estatística no contexto brasileiro. Este modelo é de relevância para o desenvolvimento de ambientes computacionais de aprendizagem inteligentes e afetivos, pois é possível concluir com evidência estatística de que a transição de emoções de alunos utilizando esses ambientes no Brasil não ocorre ao acaso. A segunda contribuição é a disponibilização do código fonte para o cálculo estatístico, exportação dos resultados e geração do gráfico da dinâmica de afetos, tornando possível que outros trabalhos utilizem esse código em suas pesquisas.

De forma geral, podemos concluir que o modelo de dinâmica de afetos desenvolvido neste trabalho corrobora com os achados propostos recentemente por [Karumbaiah et al. 2021], no qual os autores apontam que poucas pesquisas obtiveram resultados que sustentam o modelo proposto por [D'Mello and Graesser 2012]. Além disso, apesar de usarmos um ambiente de aprendizagem e um método de coleta de emoções diferentes dos que foram usados em [Karumbaiah et al. 2021], podemos notar várias transições similares no modelo do contexto brasileiro e dos Estados Unidos. Ao mesmo tempo, pode-se notar transições distintas, até mesmo com probabilidades inversas, como é o caso da ENGajamento $\rightarrow$ FRUstração.

Utilizamos o termo "no contexto brasileiro" para posicionar este trabalho em uma área ainda incipiente no Brasil. Porém, é importante destacar que a coleta de dados ocorreu com alunos de uma escola privada da região sul do país e também um ambiente de aprendizagem inteligente específico. Assim, destacamos a limitação da coleta de dados que não considerou escolas públicas, as diversas culturas ao redor do Brasil, assim como outros ambientes de aprendizagem, e sugerimos como trabalho futuro a replicação dessa pesquisa com outros perfis de estudantes brasileiros, assim como outros ambientes.

Este modelo de dinâmica de afetos no contexto brasileiro inclui várias transições significativas para o estado outro, referente a uma emoção diferente ou não identificada. Assim, apontamos como trabalho futuro a reanálise das emoções mais frequentes de alunos utilizando ambientes computacionais de aprendizagem no contexto brasileiro, pois estas emoções, assim como o modelo de dinâmica de afetos, podem diferir da literatura que conhecemos atualmente. Além disso, [Reis et al. 2018] descrevem que a personalidade dos alunos é capaz de interferir no tempo de permanência em emoções negativas. Dessa forma, acreditamos que outro trabalho futuro seria o desenvolvimento de modelos de dinâmica de afetos que consideram a personalidade dos alunos.

\section{Agradecimentos}

O presente trabalho foi realizado com apoio da Coordenação de Aperfeiçoamento de Pessoal de Nível Superior - Brasil (CAPES) - Código de Financiamento 001, da FAPERGS (Processo 17/2551-0001203-8) e do CNPq (processo 306005/2020-4). 


\section{Referências}

Andres, J. M. L. and Rodrigo, M. M. T. (2014). The incidence and persistence of affective states while playing newton's playground. In 7th IEEE international conference on humanoid, nanotechnology, information technology, communication and control, environment, and management.

Arroyo, I., Woolf, B. P., Burelson, W., Muldner, K., Rai, D., and Tai, M. (2014). A multimedia adaptive tutoring system for mathematics that addresses cognition, metacognition and affect. IJAIED, 24(4):387-426.

Azevedo, O., Morais, F., and Jaques, P. A. (2018). Exploring gamification to prevent gaming the system and help refusal in tutoring systems. In European Conference on Technology Enhanced Learning, pages 231-244. Springer.

Azevedo, R. and Aleven, V. (2013). International handbook of metacognition and learning technologies, volume 26. Springer.

Baker, R. S., D’Mello, S. K., Rodrigo, M. M. T., and Graesser, A. C. (2010). Better to be frustrated than bored: The incidence, persistence, and impact of learners' cognitiveaffective states during interactions with three different computer-based learning environments. International Journal of Human-Computer Studies, 68(4):223-241.

Baker, R. S., Moore, G. R., Wagner, A. Z., Kalka, J., Salvi, A., Karabinos, M., Ashe, C. A., and Yaron, D. (2011). The dynamics between student affect and behavior occurring outside of educational software. In International Conference on Affective Computing and Intelligent Interaction, pages 14-24. Springer.

Bosch, N. and D'Mello, S. (2013). Sequential patterns of affective states of novice programmers. In The First Workshop on AI-supported Education for Computer Science (AIEDCS 2013), pages 1-10.

Calvo, R. A. and D'Mello, S. K. (2010). Affect detection: An interdisciplinary review of models, methods, and their applications. IEEE Transactions on Affective Computing, $1(1): 18-37$.

Corrêa, A., Mello, A., Ficheman, I., and Lopes, R. (2016). Estudo sobre o impacto dos espaços interativos dos museus de ciências no processo de ensino e aprendizagem. Brazilian Symposium on Computers in Education (Simpósio Brasileiro de Informática na Educação - SBIE), 27(1):500.

Dantas, A., Melo, S., Fernandes, M., and Takahashi, E. (2015a). Aplicação para reconhecimento dinâmico de emoções em ambientes virtuais de aprendizagem. Anais dos Workshops do Congresso Brasileiro de Informática na Educação, 4(1):390.

Dantas, A., Melo, S., Moura, F., and Fernandes, M. (2015b). Reconhecimento dinâmico de emoções através de expressões faciais utilizando árvore de decisão. Brazilian Symposium on Computers in Education (Simpósio Brasileiro de Informática na Educação - SBIE), 26(1):1102.

D'Mello, S. (2013). A selective meta-analysis on the relative incidence of discrete affective states during learning with technology. Journal of Educational Psychology, 105(4):1082. 
D'Mello, S. and Calvo, R. A. (2013). Beyond the basic emotions: what should affective computing compute? In CHI'13 Extended Abstracts on Human Factors in Computing Systems, pages 2287-2294. ACM.

D’Mello, S., Graesser, A., and Taylor, R. S. (2007a). Monitoring affective trajectories during complex learning. In Proceedings of the annual meeting of the cognitive science society, volume 29 .

D'Mello, S., Picard, R. W., and Graesser, A. (2007b). Toward an affect-sensitive autotutor. IEEE Intelligent Systems, 22(4).

D'Mello, S. and Graesser, A. (2012). Dynamics of affective states during complex learning. Learning and Instruction, 22(2):145-157.

D’Mello, S., Lehman, B., Pekrun, R., and Graesser, A. (2014). Confusion can be beneficial for learning. Learning and Instruction, 29:153 - 170.

Ekman, P. (1994). All Emotions are Basic. In Davidson, R., editor, The nature of emotions: Fundamental questions, pages 15-19. Oxford University Press, Oxford.

Fredrickson, B. L. (1998). What good are positive emotions? Review of General Psycho$\log y, 2(3): 300-319$.

Gonzalez, S. M. and Tamariz, A. D. R. (2014). Integração de uma metodologia de ensino presencial de programação com um sistema tutor inteligente. Revista Brasileira de Informática na Educação, 22(02):16.

Gottardo, E. and Pimentel, A. (2018). Reconhecimento e adaptação à dinâmica de estados afetivos relacionados à aprendizagem. Brazilian Symposium on Computers in Education (Simpósio Brasileiro de Informática na Educação - SBIE), 29(1):1223.

Graesser, A. and D'Mello, S. K. (2011). Theoretical perspectives on affect and deep learning. In New perspectives on affect and learning technologies, pages 11-21. Springer.

Graesser, A. C., D’MELlO, S. K., and Strain, A. C. (2014). Emotions in advanced learning technologies. In International handbook of emotions in education, pages 483503. Routledge.

Jaques, P. A., Seffrin, H., Rubi, G., Morais, F., Ghilardi, C., Bittencourt, I. I., and Isotani, S. (2013). Rule-based expert systems to support step-by-step guidance in algebraic problem solving: The case of the tutor PAT2math. Expert Systems with Applications, 40(14):5456-5465.

Karumbaiah, S., Andres, J., Botelho, A. F., Baker, R. S., and Ocumpaugh, J. (2018). The implications of a subtle difference in the calculation of affect dynamics. In 26th International Conference for Computers in Education.

Karumbaiah, S., Baker, R. B., Ocumpaugh, J., and Andres, A. (2021). A re-analysis and synthesis of data on affect dynamics in learning. IEEE Transactions on Affective Computing.

Karumbaiah, S., Baker, R. S., and Ocumpaugh, J. (2019). The case of self-transitions in affective dynamics. In International Conference on Artificial Intelligence in Education, pages 172-181. Springer. 
Kuppens, P. (2015). It's about time: A special section on affect dynamics. Emotion Review, 7(4):297-300.

Lehman, B., Matthews, M., D’Mello, S., and Person, N. (2008). What are you feeling? investigating student affective states during expert human tutoring sessions. In International Conference on Intelligent Tutoring Systems, pages 50-59. Springer.

Marques, M., Cavalheiro, S., Foss, L., Avila, C., and Bordini, A. (2017). Uma proposta para o desenvolvimento do pensamento computacional integrado ao ensino de matemática. Brazilian Symposium on Computers in Education (Simpósio Brasileiro de Informática na Educação - SBIE), 28(1):314.

Matayoshi, J., Karumbaiah, S., et al. (2020). Adjusting the 1 statistic when self-transitions are excluded in affect dynamics. Journal of Educational Data Mining, 12(4):1-23.

Melo, S., Dantas, A., and Fernandes, M. (2017). Modelo do estudante baseado em emoções e perfis de personalidade para recomendação de estratégias pedagógicas personalizadas. Brazilian Symposium on Computers in Education (Simpósio Brasileiro de Informática na Educação - SBIE), 28(1):967.

Morais, F., da Silva, J., Reis, H., Isotani, S., and Jaques, P. (2017). Computação afetiva aplicada à educação: uma revisão sistemática das pesquisas publicadas no brasil. In Brazilian Symposium on Computers in Education (Simpósio Brasileiro de Informática na Educação-SBIE), volume 28, page 163.

Morais, F. and Jaques, P. A. (2019a). Detecção e predição de estados afetivos baseadas em mineração de dados educacionais: considerando a personalidade do aluno para aumentar a precisão da detecção. Anais dos Workshops do Congresso Brasileiro de Informática na Educação, 8(1):1052.

Morais, F. and Jaques, P. A. (2019b). Predição de emoções baseada em mineração de dados: considerando a personalidade para melhorar a detecção. In Simpósio Brasileiro de Informática na Educação (SBIE), volume 30.

Morais, F. and Jaques, P. A. (2020). Considerando personalidade e transições de emoções na detecção de emoções baseada em mineração de dados. Revista Brasileira de Informática na Educação, 28:749-775.

Morais, F. and Jaques, P. A. (2021). Does handwriting impact learning on math tutoring systems? Informatics in Education.

Morais, F., Kautzmann, T. R., Bittencourt, I. I., and Jaques, P. A. (2019). Emap-ml: A protocol of emotions and behaviors annotation for machine learning labels. In EC-TEL, pages 495-509, Netherlands. Springer.

Ocumpaugh, J. (2015). Baker rodrigo ocumpaugh monitoring protocol (bromp) 2.0 technical and training manual. New York, NY and Manila, Philippines: Teachers College, Columbia University and Ateneo Laboratory for the Learning Sciences.

Ocumpaugh, J., Andres, J. M., Baker, R., DeFalco, J., Paquette, L., Rowe, J., Mott, B., Lester, J., Georgoulas, V., Brawner, K., et al. (2017). Affect dynamics in military trainees using vmedic: From engaged concentration to boredom to confusion. In International conference on artificial intelligence in education, pages 238-249. Springer.

Pekrun, R. (2014). Emotions and learning. In Educational practices series. IEA, IBE. 
Pekrun, R. (2016). Academic emotions. Handbook of motivation at school, 2:120-144.

Reis, H., Alvares, D., Jaques, P., and Isotani, S. (2018). Analysis of permanence time in emotional states: A case study using educational software. In ITS, pages 180-190.

Scherer, K. R. (2005). What are emotions? and how can they be measured? Social Science Information, 44(4):695-729. 